\title{
Design and Implementation of Infrared (IR) Communication System
}

\author{
Suha I. Al-nassar, Haraa Raheem Hatem, Jinan N. Shehab \\ Department of Communication Engineering, Engineering College, University of Diyala \\ suha_alnassar@engineering.uodiyala.edu.iq, haraa@engineering.uodiyala.edu.iq
}

\begin{abstract}
Wireless Infrared (IR) communications system is meant to use free-space propagation of light waves as a transmission medium in near infrared band. In this paper, voice communication system is simulated and implemented by protues software using IR as a source that is established an audio communication (link to transmit and receive voices and music via infrared light). The outcome of this proposed work is to design and implementation an optical wireless system to transmit voice over a certain distance in laboratory. This system has many advantages such as is a common, inexpensive, and the transmitter or receiver can be showed to another location with least distraction.
\end{abstract}

Keywords: IR, Audio communication, Optical wireless.

Paper History: (Received: 31/10/2016; Accepted: 9/4/2017)

\section{Introduction}

Free Space Optical (FSO) system is defined that a system modulates visible or infrared (IR) beams within the atmosphere to broadcast any type of signals via an optical communication system in free space. IR radiation is basically light that cannot be seen, which shuffling it incredible for communication. The sun, light bulbs, or anything with heating system is very bright in the IR spectrum. An infrared Light Emitting Diode (LED) is a key component of an infrared system to emit the light and a photo-diode in the television or equipment to receive the light. Infrared emission technology plays an important role in popular applications, it carries a potential for enabling people with a diversity of inabilities to retrieve a list of data resources. Furthermore, Infrared technology is utilized and developed for remote control of environmental control systems, personal computers, and talking signs [1, 2, 3]. An inexpensive system through the range of lower middle class society is designed in this paper, so makeable and easy with the convenient equipment that the technical besides nontechnical person can manufacture it by themselves for their personal employment and can be used for commons conversion purpose besides for transmission of confidential data.

\section{Related Works}

In 2005, Y. Matsuya et al. designed compact technology with high-perfection audio generation. In this design using ONOFF keying and transmitted signal through an infrared LED with 1-bit A/D conversion to convert digital signal to infrared signal. Then the received infrared signal was converted into an electrical signal and reformed the waveforms by logical system and converted them to analog signals with a simple low pass filter [2]. In 2011, Beaver et al. studied the effect distance of operation in IR communication system design and these distances alternation with the intensity of the IR which is emitted by the LED on the transmitter. Also using a single jumper to control on the number of LED that emitting light. Where the maximum current of LED is taken value $100 \mathrm{~mA}$, the configuration of jumper limits the current through the LED to approximately $70 \mathrm{~mA}$ [4]. Also in 2014, Jiamn Zhou et al. designed IR communication system to transmit the voice and digital signal with short distance [5].

\section{Infrared Wireless Communication System} Wireless communication allows information to be transmit between two devices without using wire or cable. The data is being transmitted and received using electromagnetic radiation, the electromagnetic spectrum orders electromagnetic energy according to wavelength or frequency, the electromagnetic spectrum ranged from energy waves having Extremely Low Frequency (ELF) to energy waves having much higher frequency, e.g. $\mathrm{X}$ rays. Infrared is an electromagnetic radiation has a wavelength longer than that of visible light but shorter than radio waves and has wavelength between $(750 \mathrm{~nm}-1 \mathrm{~mm})$ as shown in Figure 1.

Infrared LEDS are classified into Near Infrared (NIR) and Far Infrared (FIR). In this paper (NIR) is our interest, it is divided into two bands the long wave and short wave (NIR), the determination of 
type depend on how the film and card digital compact disk (cd) cameras react with it. So the used part of the infrared spectrum in laser communication system is divided into various bands based on the type of the light sources, transmitting labsorbing materials(fibers) and detectors.

IR communication system consists of three main parts transmitter circuit, medium propagation (IR) and receiver circuit as shown in Figure 2. In this paper, short distance transmission of voice is realized by the design and achievement of infrared communication link. The modem part of this design is carried out by the phase locked circuit consisted of TDA2002 [2, 3, 4, 5, 6].

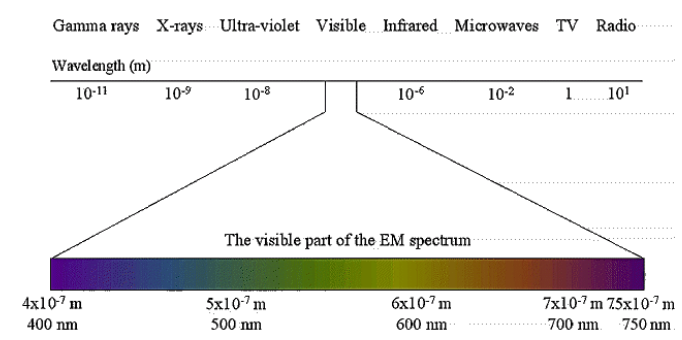

Figure 1: The electromagnetic spectrum

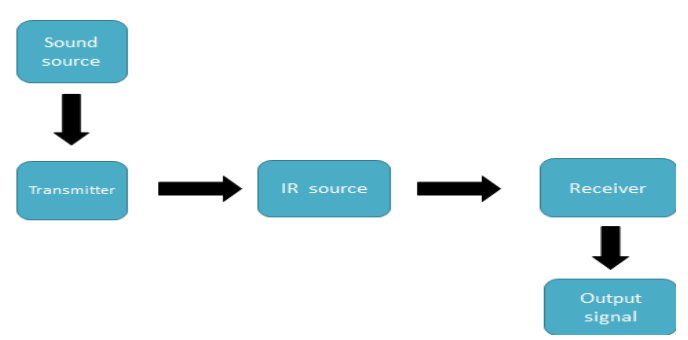

Figure 2: The block diagram of IR communication system

\section{Methodology for Proposed System}

To achieve this proposed work with low cost, a number of related circuit diagram has been downloaded from different websites. After confirmation, an appropriate circuit has been chosen for modification according to the available equipment in our country for preparing a low cost design system. The proposed work was modified in such way so that popular people of Iraq can make it effectively with moderate hardware here. The proposed circuit was structured on a bread board. Each of the transmitter and the receiver circuits using 9V battery as a power supply which are built around TDA 2002, This work done at Communication Department, Engineering College, University of Diyala.

Among different stage test, firstly the transmitter was tested using IR available in anywhere in the market. Electronic equipment have been modified in many stages according to the neediness and availability in our country so that get appropriate output with the possible minimum cost. After getting the results, the range as well as the efficiency of the system was verified. Figure 3 shows the methodology for proposed system which display the flow chart of this work.

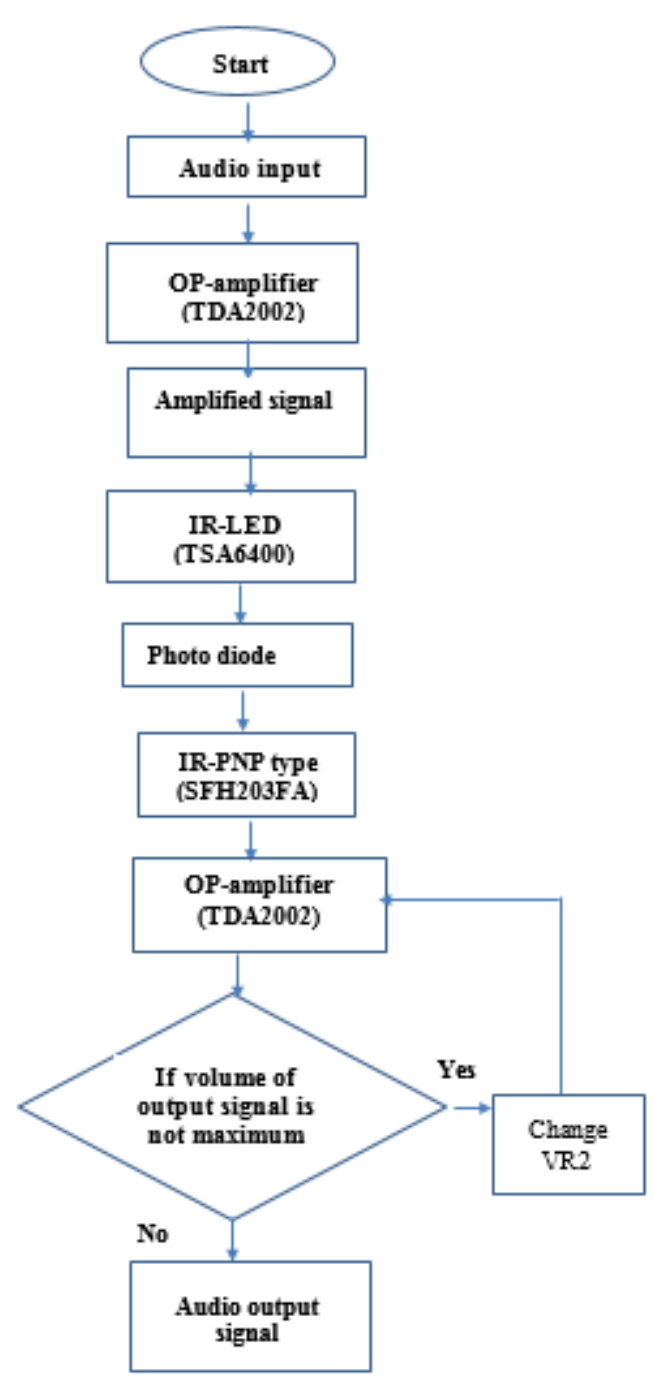

Figure 3: The Flowchart of the presented work

\subsection{Transmitter Circuit}

Figure 4 shows the simulated circuit using proteus software for the transmitter circuit utilizing infrared (IR) emitter LED as the light sources. In this circuit using the public generator IC (TDA2002) to generate a ceaseless musical tone. To get the greatest range, IC (TDA2002) is fed to the infrared (IR) driver. In this stage, IC (TDA2002) is created the red LED flashes of musical tones, indicating modulation process.

The electrical signal which generated from IC (TDA2002) is transformed into an invisible infrared light signal by the infrared light emitting diode (IR LED) type (TSAL 6400). A laser diode (LED1) has maximum operating 
current of $45 \mathrm{~mA}$ and voltage of about $3.3 \mathrm{~V}$ $\mathrm{DC}$, this maximum operating is utilized to transmit the audio signal. The division of voltage network formed by $\mathrm{R} 1$, and $\mathrm{R} 2$, also it protects the current and the voltage for the laser diode in the safe region.VR1 $(100 \mathrm{~K} \Omega)$ is utilized to vary the scale of the input audio signal. The audio input signal (Vin) is taken from the preamplifier output of the music system. VR2 and Capacitor C6 are used to change the gain of the IC (TDA2002). Table 1 represents the necessary equipment for preparing transmitter circuit.

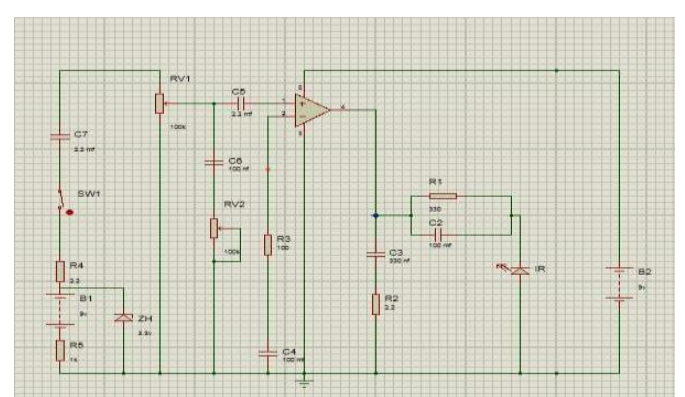

Figure 4 : Transmitter circuit by using Proteus software

Table 1 The important components for transmitter circuit.

\begin{tabular}{|c|c|c|}
\hline Resistors & Capacitors & Other \\
\hline $\mathrm{R} 1=330 \Omega$ & $\mathrm{C} 1=100 \mu \mathrm{F}$ & $\begin{array}{c}\text { IR-LED } \\
\text { TSAL } \\
6400\end{array}$ \\
\hline $\mathrm{R} 2=2.2 \Omega$ & $\mathrm{C} 2=100 \mu \mathrm{F}$ & $\mathrm{ZH}=3.3 \mathrm{v}$ \\
\hline $\mathrm{R} 3=100 \Omega$ & $\mathrm{C} 3=330 n \mathrm{~F}$ & $\begin{array}{c}\text { Battery } \\
=9 \mathrm{v}\end{array}$ \\
\hline $\mathrm{R} 4=2.2 \Omega$ & $\mathrm{C} 4=100 \mu \mathrm{F}$ & $\begin{array}{c}\mathrm{IC} \\
\text { (TDA2002) }\end{array}$ \\
\hline $\mathrm{R} 5=1 \mathrm{k} \Omega$ & $\mathrm{C} 5=2.2 \mu \mathrm{F}$ & \\
\hline $\begin{array}{c}\mathrm{VR} 1=100 \\
\mathrm{~K} \Omega\end{array}$ & $\mathrm{C} 6=100 \mathrm{nF}$ & \\
\hline $\mathrm{VR} 2=100$ & $\mathrm{C} 7=2.2 \mu \mathrm{F}$ & \\
$\Omega$ & & \\
\hline
\end{tabular}

\subsection{Receiver Circuit}

Figure 5 shows the simulated circuit of the receiver circuit by using electronic transistor IR-PNP type (SFH203FA) to receive the signaling from IR-LED and then it come out from the speaker has a low amplitude voltage with 9V battery inside the receiver circuit. Using photodiode in the receiver circuit to convert the infrared light signal to an electrical signal. The sound recording infrared (IR) receiver uses audio frequency amplifier and public operational amplifier IC (TDA2002) along with photodiode and some special components. Via IR- LED the melody which be generated by IC (TDA2002) is transmitted, after that it received by photodiode and fed to pin 2 of IC (TDA2002). The benefit of variable resistanceVR1 is to change the gain. The output signal is fed to IC (TDA2002) through variable resistor (VR2) and capacitor. Finally, the largest electrical signal which carry by the speaker that convert electrical energy into sound energy. The produced signal is responded via the receiver loudspeaker. Also to adjust the volume of loudspeaker occurred by using variable resistor VR2. For maximum sound transmission desire to be oriented towards and must collision the infrared (IR) PNP transistor at the receiver. Table 2 represents the necessary equipment to design receiver circuit.

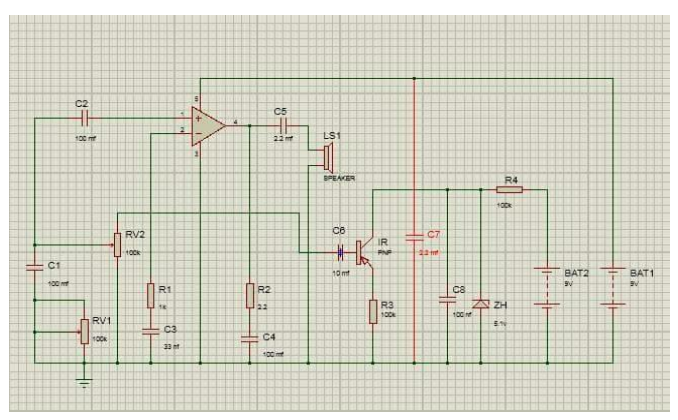

Figure 5: Receiver circuit by using Proteus software

Table 2 The important components for receiver circuit.

\begin{tabular}{|c|c|c|}
\hline Resistors & Capacitors & Other \\
\hline $\mathrm{R} 1=1 \mathrm{~K} \Omega$ & $\mathrm{C} 1=100 \mu \mathrm{F}$ & $\begin{array}{c}\text { IC- TDA } \\
2002\end{array}$ \\
\hline $\mathrm{R} 2=2.2 \Omega$ & $\mathrm{C} 2=100 \mu \mathrm{F}$ & $\begin{array}{c}\text { IR-PNP } \\
\text { SFH203FA }\end{array}$ \\
\hline $\mathrm{R} 3=100 \mathrm{~K} \Omega$ & $\mathrm{C} 3=33 n \mathrm{~F}$ & $\mathrm{ZH}=5.1 \mathrm{v}$ \\
\hline $\mathrm{R} 4=100 \Omega$ & $\mathrm{C} 4=100 \mu \mathrm{F}$ & $\begin{array}{c}\text { Battery }=9 \\
\text { v }\end{array}$ \\
\hline $\mathrm{VR} 1=100 \mathrm{~K} \Omega$ & $\mathrm{C} 5=2.2 \mu \mathrm{F}$ & \\
\hline $\mathrm{VR} 2=100 \mathrm{~K} \Omega$ & $\mathrm{C6}=10 \mu \mathrm{F}$ & \\
\hline & $\mathrm{C} 7=2.2 \mu \mathrm{F}$ & \\
\hline & $\mathrm{C} 8=100 n \mathrm{~F}$ & \\
\hline
\end{tabular}

\section{Design and Construction of the Proposed Circuit}

Figure 6 shows the construction of transmitter circuit with components. The transmitter board contained many important components such as a dynamic microphone and an IR source with $210 \mathrm{mw}$ at opposite ends. The input signal is from mobile or radio, so the output signal will amplifier. Thus the necessary part of circuit is amplifier. These signals are received with the optical condenser microphone and transferred into electrical distinction, that are amplified by the operational amplifier, using of IC- TDA2002 in the inverting mode with a single supply occurred by divider network of resistor. It's 
gain of IC- TDA2002 can be set varying the feedback through the resistor R5, here the output signal of IC is further amplified via the push-pull amplifier using electronic transistor IR-PN (SFH203FA) pair, the mapping of R2 is feedback resistance with $\mathrm{R} 1$ and $\mathrm{C} 1$ to connected IC- TDA2002. Pin 2 in ICTDA2002 is connected with resistance (VR1) through $\mathrm{O} / \mathrm{P}$ of (transistor). To inhibit tune the microphone place near the circuit with the shield wire. The output signal of the amplifier is taken from emitter of two transistors with a filter C5. The same process continues in the second amplifier.

Figure 7 shows the construction of receiver circuit, that use a PNP transistor to receive the signal from IR and then it come out from the speaker has a low amplitude voltage. The receiver used Infrared modules IR-signal which reached from the transmitter and is sensed by the detector and its output PIN 1 goes low and switched LED. At Pin 14, the IC- TDA2002 is worked on clock pulse which receives to infrared modules. As mentioned the output signal from IC is used for lighting, LED-1 this indicating to presence of signal, i.e. LED is switched 'ON. Oppositely when no signal is available, output of sensor module goes high and LED is switched 'OFF'. This makes the LED to switch 'ON' when the appliance at first pulse and 'OFF' when the device at its second pulse

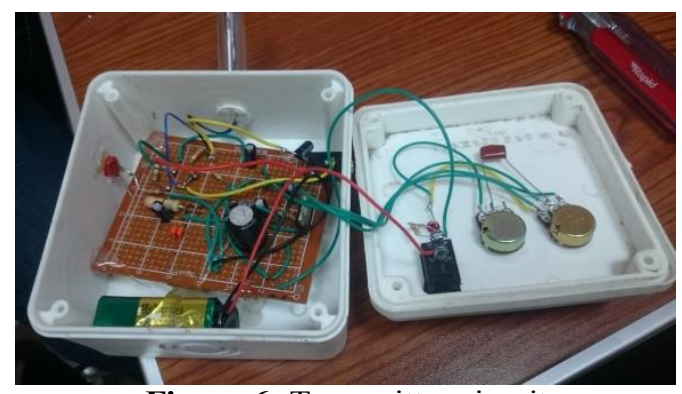

Figure 6: Transmitter circuit

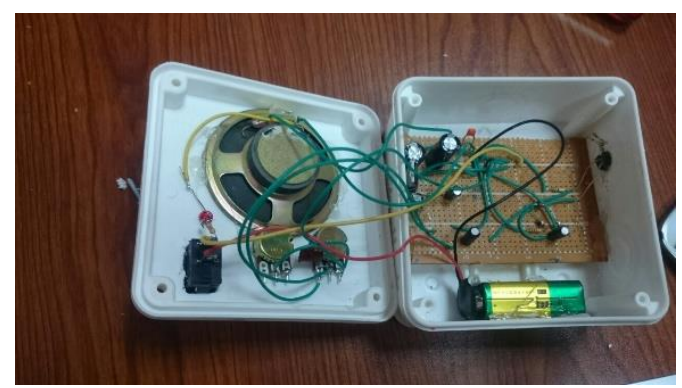

Figure 7: Receiver circuit

\section{Results}

Figure 8 shows the complete design of optical wireless audio system. The supplying voltage from battery for each IR transmitter and receiver is $(9 \mathrm{~V})$. Transmitter utilizes light as a data common carrier. To produce a series of audio signal encoding the transmitter via modulation frequency. Then the signal light will be received by the receiver, the modulated and enforced signals can be sent by amplifier where the music can be heard.

The infrared (IR) radiation using as the light sources in transmitter circuit. It utilizes general melody generator community type (IC TDA2002) which can generate a continuous musical tone. In receiver circuit, the ICTDA2002 product is fed to the infrared (IR) driver stage which built cross transistor PNP in order to get the maximum range. Here, the LED flashes indicating to musical tones which generated by IC-TDA2002, this meant to start the modulation physical process. With the infrared light emitting diode (IR- LED) the electrical signal generator from TDA2002 which is transferred into an invisible infrared light signal. For maximum sound transmission requests orientation course and must hitting the infrared (IR) photodiode at the receiver electric circuit. In this circuit the photodiode converts this invisible infrared light signal into an electrical signal. The infrared (IR) audio system receiver uses audio frequency amplifier in IC-TDA2002 and PNP photodiode transistor and some distinct components. The melody generated by IC- TDA2002 is transmitted through IR- LED, and then received by photodiode. It's gain can be varied using a variable resistor VR1.

The main result of this proposed work is to design and implementation an optical wireless system to transmit voice over a certain distance in laboratory with many advantages such as is a common, inexpensive.

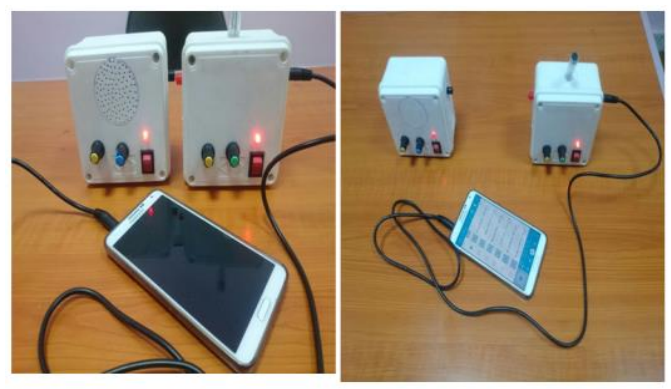

Figure 8: The circuit working of IRcommunication system 


\section{Conclusions}

This paper is focusing on transmitting audio signal (mobile or radio) from the transmitter ending to the receiver ending using the infrared light radiation equipment, this design is called the optical wireless audio system, The designing in this paper devoted on the development of the conventional infrared radiation communicating by increasing the transmission distance and the effective signal coverage region, likewise this system has unique advantages such as minimal effort with low cost, high speed communication and almost no limitations of bandwidth range.

This project can be made and used successfully at conference room, political assembly, and class room and for general conversation between two houses. So the lowest cost of this design in our country is better to use for general conversation with neighbors where confidentiality is a prime issue.

\section{References}

[1]. Je®rey B. Carruthers, , Wireless Infrared Communications, Wiley Encyclopedia of Telecommunications, 2002.

[2]. N. R. Mohamad, A. A. Wakiran, M. F. Iswandi, A. Salleh, S. A. M. J. Yunus, F. Sallehuddin, N. F. Azmi and S. A. M. Chach, Development of Optical Wireless Audio System using Infrared Light Communication, IOSR Journal of Electronics and Communication Engineering (IOSR-JECE) ,Vol. 8, Issue 6 (Nov. - Dec. 2013), 65-69. www.iosrjournals.org .

[3]. Tee Kok Sen, Wireless Optical Voice Communication Link, report for the word of. Bachelore of electronic engineering "Telecommunication Electronic", 2008 .

[4]. A. Beaver, M. Hannon, and A. Jalilzadeh, Infrared FM Audio Communication System, 2011.

[5]. Jiamn Zhou, Rener Yang and Xuyan Ni, Design and implementation of Infrared communication system, 496-500, (2014), 2091-2094.

[6]. Joseph M. Kahn, Member, and John R. BAR, Wireless Infrared Communications, 0018-9219/97 , IEEE,( 1997). 